\title{
Stents en fístulas esofagogástricas posquirúrgicas: in medio virtus
}

\section{Stents for treating postoperative esophagogastric fistulas: in medio virtus}

Rodrigo Castaño-Llano..$^{*}$



De acuerdo con el Grupo de Consenso sobre Complicaciones de la Esofagectomía, una fuga anastomótica es un "defecto gastrointestinal de espesor total que afecta al esófago, la anastomosis, la línea de grapas o el conducto" ${ }^{(1)}$. Las fugas anastomóticas después de la cirugía se han asociado a tasas más altas de morbimortalidad, especialmente si hay un retraso de más de 48 horas en el diagnóstico ${ }^{(2,3)}$. El fracaso en el rescate de una fuga esofágica es la principal causa de mortalidad en estos pacientes. El drenaje adecuado, la reintervención con colgajos pleurales o diafragmáticos y la derivación (esofagostomía) son opciones de tratamiento para las fugas graves no controladas. Recientemente, se ha despertado el interés por las opciones mínimamente invasivas para tratar las fugas anastomóticas ${ }^{(4)}$, incluida la colocación de endoprótesis esofágicas, que han demostrado ser eficaces en el tratamiento inicial de las fugas anastomóticas esofágicas y pueden ofrecer comorbilidades bajas, con resultados comparables a las de otros tratamientos ${ }^{(5-9)}$.

Las endoprótesis se colocan por vía endoscópica para evitar o tratar las complicaciones quirúrgicas y ofrecer una menor duración de la estancia a los pacientes ${ }^{(6)}$. Incluso con una buena técnica de colocación de las endoprótesis ${ }^{(10)}$, siguen produciéndose complicaciones. La migración del stent, el dolor del paciente, la erosión del tejido vecino y la fuga persistente son algunas de las complicaciones más comunes. El tipo de endoprótesis y el método de colocación pueden variar debido a la disponibilidad local, las preferencias del médico y las características del paciente, lo que puede contribuir a los diferentes riesgos de complicación. Se cree que hay varios factores que contribuyen al fracaso de la endoprótesis, como una mala selección del paciente, un diámetro incorrecto de la endoprótesis, una mayor duración de la estancia de la endoprótesis, una actividad funcional baja antes de la cirugía, el desarrollo de una fístula esofágica, la exposición previa a la quimioterapia, el retraso en el diagnóstico de la fuga 48 horas después de producirse, una mayor edad, el aumento del tamaño de la fístula y las comorbilidades graves ${ }^{(6,7,9)}$.

En este número se presenta el estudio de Ovalle y colaboradores, de carácter unicéntrico y retrospectivo, quienes durante 11 años estudiaron a 11 pacientes con fugas anastomóticas esofagogástricas posquirúrgicas a quienes se les colocó 14 stents y se evaluaron los desenlaces técnicos y clínicos. El éxito técnico fue del $100 \%$ y el éxito clínico se dio en el 72,7 \% con un cierre de las fístulas del 63,5\% (7/11 pacientes). Las complicaciones fueron del $27,7 \%(3 / 11)$ y se relacionaron solo con la migración del stent ( 1 caso con 3 migraciones y 3 posturas de stent).

Al hacer en análisis retrospectivo de las publicaciones del último decenio en el tratamiento de las fugas esofagogástricas con endoprótesis, se produjeron ciertos ajus- 
tes de este procedimiento para el manejo de estas, como el aumento en el diámetro de los stents, recubrimientos totales frente a los parciales y una mayor disponibilidad y experticia al momento de su colocación ${ }^{(10)}$. Con respecto a los diámetros de los stents, se realizó un estudio clínico en el que se probaron diámetros mayores (megastents) para el manejo de este tipo de complicaciones con una tendencia a una menor tasa de migración en comparación con controles históricos tratados con un stent convencional ${ }^{(11)}$.

El presente estudio, al igual que las diferentes publicaciones, muestra grandes diferencias en el diagnóstico y tratamiento de las fugas esofagogástricas; se describen diferentes métodos diagnósticos, y en la actual serie el esofagograma es el más frecuente (7/11 pacientes); no se enfatiza en el tamaño del defecto, hay distintas indicaciones para la colocación de las endoprótesis, diferentes tiempos al momento de colocar el stent y el diagnóstico inicial de fuga, diversos tipos de endoprótesis (tamaño, cubrimiento parcial o total), el uso de otras técnicas endoscópicas como el EndoVac o en este caso el clip OVESCO, y el protocolo de manejo postratamiento referente a los estudios radiológicos, que pueden ir de una simple radiografía a una tomografía.

Estos interrogantes no pueden ser resueltos con ninguna serie retrospectiva y difícilmente se puede poner en marcha un estudio aleatorizado comparando el uso del stent frente a otro grupo sin terapia endoscópica, por consideraciones éticas. Actualmente, los estudios se orientan hacia definir el papel del stent frente a otras opciones endoscópicas como los sellantes de fibrina, los endoclips o las terapias de vacío como el EndoVac o las combinaciones de estas terapias, como el caso de la serie, tratado exitosamente con OVESCO más stent.
Es necesario un protocolo estandarizado de diagnóstico y tratamiento en pacientes clínicamente sospechosos de fugas luego de la esofagectomía o gastrectomía, lo que permitiría obtener mejores resultados. Recientemente, hemos presentado en el Congreso Nacional de Cirugía nuestra experiencia con el manejo de 22 pacientes con fístulas esofágicas luego de la esofagectomía y el ascenso gástrico en el cuello, con un éxito técnico del $100 \%$ y un cierre de las fístulas del $64 \%{ }^{(12)}$, la propuesta de manejo se recoge en la Figura 1.

Se ha sugerido que el momento óptimo para la colocación del stent es dentro de las primeras 24 horas después del diagnóstico de la fuga, con el fin de limitar las complicaciones infecciosas y favorecer la cicatrización ${ }^{(13)}$. Todavía no se ha establecido un consenso sobre el tiempo óptimo de retiro de la endoprótesis para lograr el cierre de una fuga esofágica. En estudios recientes, el tiempo necesario varía entre 4 y 12 semanas $^{(14)}$.

Mientras que los pacientes clínicamente estables con pequeñas fugas contenidas pueden tratarse de forma conservadora con antibióticos intravenosos y un posible drenaje percutáneo, los pacientes con contaminación mediastínica y pleural pueden ahora a menudo salvar su conducto utilizando stents esofágicos, terapia de vacío endoluminal $\mathrm{u}$ otras suturas y clips endoluminales. El beneficio de la nutrición enteral continuada con la colocación de la endoprótesis endoluminal debe sopesarse frente a las mayores tasas de complicaciones y migración de la endoprótesis en comparación con el EndoVac. Aunque el EndoVac ha mostrado resultados prometedores en el cierre de fugas anastomóticas, se justifica la realización de estudios adicionales sobre su uso en pacientes con fugas complejas. A medida que la medicina siga evolucionando, se encontrarán formas

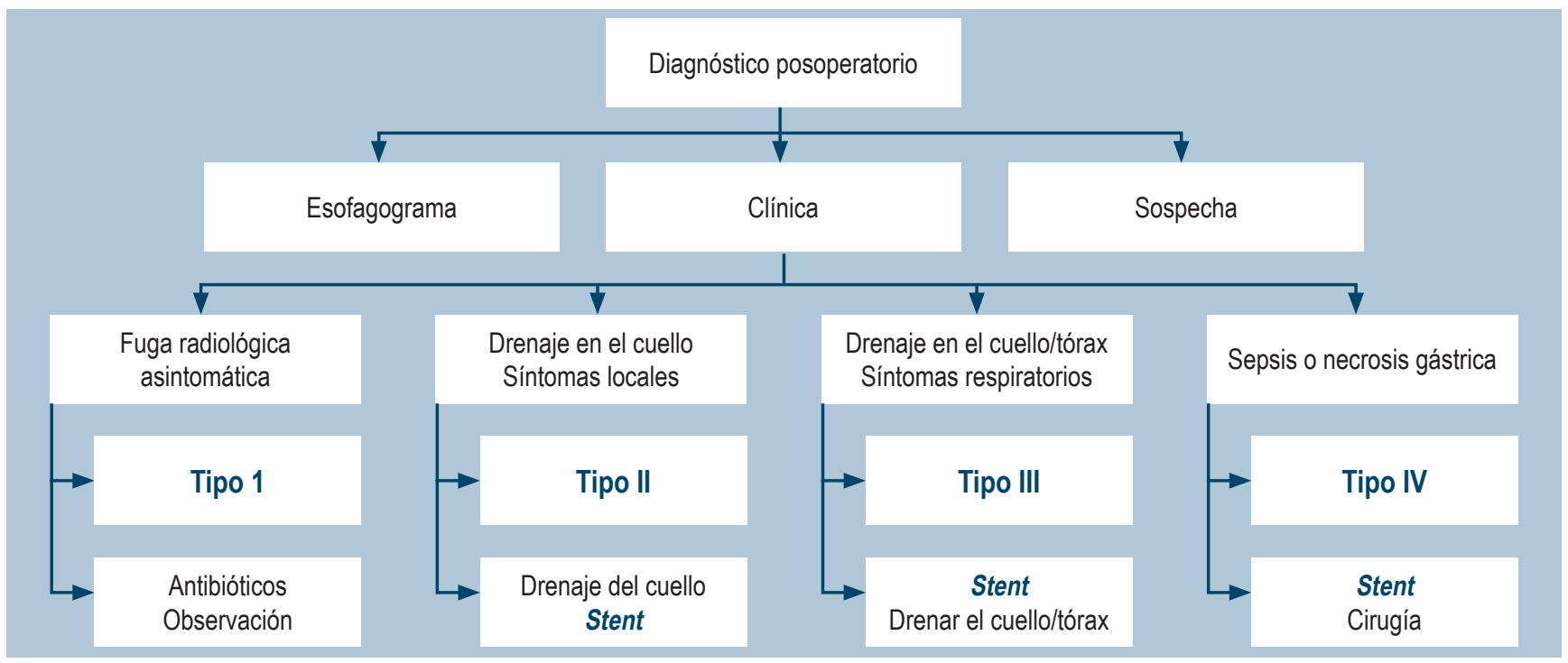

Figura 1. Algoritmo para el diagnóstico y manejo de las fístulas esofagogástricas posoperatorias. 
más novedosas de abordar esta complicación y algún día se evitarán las fugas anastomóticas por completo.

Las limitaciones del estudio incluyen su naturaleza retrospectiva, con resultados que reportan datos de un centro terciario y único, con un posible sesgo de selección que puede impedir la generalización a la práctica diaria, así como el pequeño número y la población heterogénea de pacientes. Sin embargo, solo refleja a los pacientes con posoperatorio de fugas esofagogástricas y aborda los factores clínicos y endoscópicos asociados con la resolución endoscópica de las fugas. Como corolario final y retomando el título del escrito, la virtud o el éxito (in medio virtus) en el manejo de estos complejos casos está en la juiciosa selección del paciente, del stent u otros medios endoscópicos para lograr, como en la presente serie de Ovalle y colaboradores, unos buenos resultados.

\section{REFERENCIAS}

1. Low DE, Alderson D, Cecconello I, Chang AC, Darling GE, D'Journo XB, Griffin SM, Hölscher AH, Hofstetter WL, Jobe BA, Kitagawa Y, Kucharczuk JC, Law SY, Lerut TE, Maynard N, Pera M, Peters JH, Pramesh CS, Reynolds JV, Smithers BM, van Lanschot JJ. International Consensus on Standardization of Data Collection for Complications Associated With Esophagectomy: Esophagectomy Complications Consensus Group (ECCG). Ann Surg. 2015;262(2):286-94. https://doi.org/10.1097/SLA.0000000000001098

2. Grimminger PP, Goense L, Gockel I, Bergeat D, Bertheuil $\mathrm{N}$, Chandramohan SM, Chen KN, Chon SH, Denis C, Goh KL, Gronnier C, Liu JF, Meunier B, Nafteux P, Pirchi ED, Schiesser M, Thieme R, Wu A, Wu PC, Buttar N, Chang AC. Diagnosis, assessment, and management of surgical complications following esophagectomy. Ann N Y Acad Sci. 2018;1434(1):254-273. https://doi.org/10.1111/nyas. 13920

3. Yeung JC. Management of Complications After Esophagectomy. Thorac Surg Clin. 2020;30(3):359-366. https://doi.org/10.1016/j.thorsurg.2020.04.002

4. Zhong L, Zhong J, Tan Z, Wei Y, Su X, Wen Z, Rong T, Hu Y, Luo K. An Approach to Accelerate Healing and Shorten the Hospital Stay of Patients With Anastomotic Leakage After Esophagectomy: An Explorative Study of Systematic Endoscopic Intervention. Front Oncol. 2021;11:657955. https://doi.org/10.3389/fonc.2021.657955

5. Liesenfeld LF, Schmidt T, Zhang-Hagenlocher C, Sauer P, Diener MK, Müller-Stich BP, Hackert T, Büchler MW, Schaible A. Self-expanding Metal Stents for Anastomotic Leaks After Upper Gastrointestinal Cancer Surgery. J Surg Res. 2021;267:516-526. https://doi.org/10.1016/j.jss.2021.06.007

6. Carter TS, Philips P, Egger M, Scoggins C, Martin RCG 2nd. Outcomes of Esophageal Stent Therapy for the Management of Anastomotic Leaks. Ann Surg Oncol. 2021. https://doi.org/10.1245/s10434-021-09669-6

7. Smith EA, Daly SC, Smith B, Hinojosa M, Nguyen NT. The Role of Endoscopic Stent in Management of Postesophagectomy Leaks. Am Surg. 2020;86(10): 1411-1417. https://doi.org/10.1177/0003134820964495
8. Kamarajah SK, Bundred J, Spence G, Kennedy A, Dasari BVM, Griffiths EA. Critical Appraisal of the Impact of Oesophageal Stents in the Management of Oesophageal Anastomotic Leaks and Benign Oesophageal Perforations: An Updated Systematic Review. World J Surg. 2020;44(4):1173-1189. https://doi.org/10.1007/s00268-019-05259-6

9. Plum PS, Herbold T, Berlth F, Christ H, Alakus H, Bludau M, Chang DH, Bruns CJ, Hölscher AH, Chon SH. Outcome of Self-Expanding Metal Stents in the Treatment of Anastomotic Leaks After Ivor Lewis Esophagectomy. World J Surg. 2019;43(3):862-869. https://doi.org/10.1007/s00268-018-4832-2

10. Castaño $R$. Técnicas en stents gastrointestinales endoscópicos: cómo, cuándo, manejo de complicaciones, selección del stent y costos. Rev Colomb Gastroenterol. 2012;27(1):32-44.

11. Castaño R, Álvarez O, Lopera J, Ruiz M, Rojas A, Alvarez A, Ruiz LM, Restrepo D. Desarrollo e implementación de un nuevo diseño de stent de nitinol para el manejo de las estenosis y fístulas benignas del tracto digestivo. Rev Colomb Gastroenterol. 2015;30(3):261-72.

12. Castaño R, Álvarez O, Jaramillo R, Palacios L, Calvo V. Efectividad en el manejo endoscópico con stents cubiertos de las fístulas en cuello post esofagectomías por malignidad. Bogotá, Semana Quirúrgica Nacional; agosto 18-21, 2019.

13. Huh CW, Kim JS, Choi HH, Lee JI, Ji JS, Kim BW, Choi H. Treatment of benign perforations and leaks of the esophagus: factors associated with success after stent placement. Surg Endosc. 2018;32(8):3646-3651. https://doi.org/10.1007/s00464-018-6096-1

14. Iglesias Jorquera E, Egea Valenzuela J, Serrano Jiménez A, Carrilero Zaragoza G, Ortega Sabater A, Sánchez Velasco E, Ruiz de Angulo D, Munitiz V, Parrilla P, Alberca de Las Parras F. Endoscopic treatment of postoperative esophagogastric leaks with fully covered self-expanding metal stents. Rev Esp Enferm Dig. 2021;113(1):14-22. https://doi.org/10.17235/reed.2020.6821/2019 